\title{
Requirements Development Issues for Advanced Life Support Systems: Solid Waste Management
}

\author{
Julie A. Levri and John W. Fisher \\ NASA Ames Research Center \\ Michael P. Alazraki \\ Lockheed Martin Space Operations \\ John A. Hogan
}

New Jersey NASA Specialized Center of Research and Training

Copyright $(2002$ Society of Automotive Engineers, Inc.

\begin{abstract}
Long duration missions pose substantial new challenges for solid waste management in Advanced Life Support (ALS) systems. These possibly include storing large volumes of waste material in a safe manner, rendering wastes stable or sterilized for extended periods of time, and/or processing wastes for recovery of vital resources. This is further complicated because future missions remain ill-defined with respect to waste stream quantity, composition and generation schedule. Without definitive knowledge of this information, development of requirements is hampered. Additionally, even if waste streams were well characterized, other operational and processing needs require clarification (e.g. resource recovery requirements, planetary protection constraints). Therefore, the development of solid waste management (SWM) subsystem requirements for long duration space missions is an inherently uncertain, complex and iterative process.
\end{abstract}

The intent of this paper is to address some of the difficulties in writing requirements for missions that are not completely defined. This paper discusses an approach and motivation for ALS SWM requirements development, the characteristics of effective requirements, and the presence of those characteristics in requirements that are developed for uncertain missions. Associated drivers for life support system technological capability are also presented. A general means of requirements forecasting is discussed, including successive modification of requirements and the need to consider requirements integration among subsystems.

\section{INTRODUCTION}

A group of researchers, referred to as the ALS Solid Waste Management Working Group has developed an approach for focusing research and technology development (R\&TD) within the area of SWM of the ALS Program. By focusing efforts, the group hopes to identify R\&TD priorities that will promote life support technological readiness for future human-rated missions.

The ALS Program develops life support technologies for both current and future long-duration human space missions. Attributes of the current long-duration mission, the International Space Station (ISS), are well known, thereby simplifying identification of beneficial life support system R\&TD. However, the range of possible future long-duration missions is broad, resulting an even broader range of relevant life support technologies. Because the ALS Program budget is finite, it is sensible to focus the range of possible relevant life support technologies to a manageable level, without inadvertently eliminating options that offer considerable advantages.

The approach that the ALS SWM Working Group has taken to focus R\&TD efforts is depicted in Figure 1, The ALS SWM Technology Selection Process. Each block in Figure 1 is provided with an identification number in the upper right-hand corner. Standard process diagram shapes are used to depict starting and ending points (ovals), inputs (parallelograms), processes (rectangles), and decisions (diamonds). To date, progress has been made on blocks 1 through 10 of the ALS SWM Technology Selection Process. The Advanced Life Support Solid Waste Management Research and Technology Development Plan (Alazraki, 2001) elucidates that progress and explains the Technology Selection Process in further detail. In this paper, a brief description is provided of the steps leading up to the point where technology attributes must be matched against requirements (block 11). 


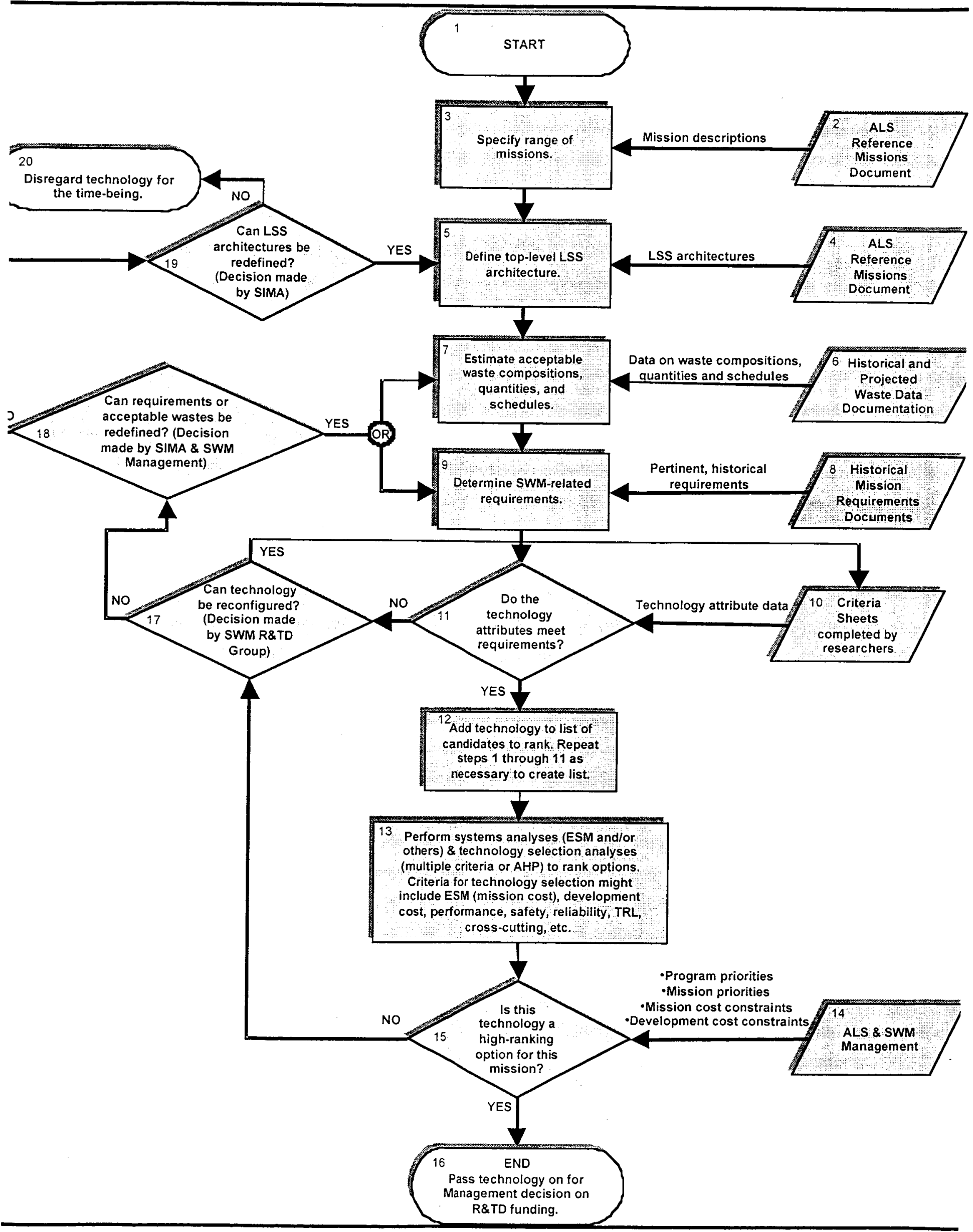

Figure 1. The ALS SWM Technology Selection Process 
The first nine blocks on Figure 1 are oriented toward identifying a set of requirements for selected missions. It should be noted that input from the ALS SWM Working Group is inherent in each of the input blocks (parallelograms) shown on the diagram. Block 10 signifies the collection of information from ALS SWM researchers and technology developers for identifying 1) what the ALS Program currently has available in terms of SWM technologies, 2) the development potential of those technologies, and 3) other pertinent information about those technologies.

Blocks 2 through 5 comprise the first step of requirements identification, which is specification of a reasonable range of possible future missions and their top-level life support system (LSS) architectures. Currently, the missions that are depicted in the ALS Reference Missions Document (RMD) (Stafford et al., 2001) are used for this purpose. The missions identified in the RMD provide a reasonable and useful range of mission types in terms of location, duration and top-level LSS architecture. RMD missions include the ISS, the Mars Dual Lander Mission and an Evolved Mars Base. As the efforts of the ALS SWM Working Group progress, alternative missions (from the JSC Exploration Office) and top-level LSS architectures (from the ALS Program), that are not currently depicted in the RMD, may be defined for use in the Technology Selection Process. Similarly, because the RMD can not capture all reasonable LSS architectures for a mission, if a particular technology does not fall into the scope of a mission in the RMD, researchers and technology developers may propose alternative missions and LSS architectures. Such alternatives would then be judged by ALS management for their relevance to the ALS Program.

Blocks 6 and 7 represent the process of identifying likely waste stream quantities, compositions and generation schedules. For future missions, estimations of solid waste generation rates must be based partially upon data from historical missions and partially on projections of waste generation as a function of the assumed top-level LSS architecture. For example, if it is expected that a portion of the crew's diet in the Mars Dual Lander mission will be supplied by salad crops, a reasonable range of inedible biomass generation rates may be incorporated into the projected waste stream.

Once missions, general LSS architectures and projected waste streams have been defined, top-level, missionspecific requirements may be developed. This process is represented with blocks 8 and 9 in Figure 1 . Requirements development is a critical step in determining the SWM R\&TD needs in the ALS Program.

This paper is the second in a set of three papers that describe the reasoning and process of developing requirements for the missions of interest to the ALS
SWM Group. The first paper in the series (Hogan et al., 2002) provides a general discussion of the critical obstacles and possibilities associated with solid waste management in long-duration human missions. The third paper in the series (Alazraki et al., 2002) provides several examples of requirements that the SWM Working Group is currently considering, which have considerable influence on the technologies needed for life support systems. The intent of this paper is to tie the first and third papers by addressing some of the difficulties in writing requirements for missions that are not completely defined.

It is important to note that this paper, as well as ALS SWM R\&TD Plan, is written from the perspective of writing requirements for improved R\&TD selection for Solid Waste Management. Although this type of approach might eventually be modified for other areas of ALS (e.g. Air, Water, Food, Biomass, and Thermal) or for a life support top-level approach, this has not yet been attempted.

\section{THE MOTIVATION FOR REQUIREMENTS DEVELOPMENT}

Typically, mission requirements are developed in order to specify needs for a specific, well-defined mission. Alternatively, the SWM Working Group is projecting possible mission requirements to determine the R\&TD necessary in order to be well-prepared for future missions. Several motivations for this effort are discussed below.

PROVIDING R\&TD GUIDELINES - The development of top-level requirements can better define goals for researchers and technology developers. A reasoned, structured set of program needs can help researchers to identify areas of interest for technology development. At the same time, such a set of needs can spark thought and discussion about what needs might be missing from that set, resulting in continuous refinement of ALS SWM requirements.

MATCHING PROGRAM NEEDS TO PROGRAM RESOURCES - By comparing Program needs with available resources, R\&TD gaps can be identified and resolved. Blocks 9,10 and 11 on Figure 1 correspond to identifying ALS Program R\&TD needs, identifying available ALS SWM technologies (resources), and comparing those two features, respectively.

Comprehension of what technologies the ALS SWM Program is currently developing requires that researchers and technology developers explain the purpose and expectations of their work. In order to make decisions about Program priorities, system analysts and managers must be able to understand and apply such information. To facilitate this interaction, an electronic 
"criteria" form has been developed (Levri et al., 2001). It is intended that researchers and technology developers complete the form in order to convey information about current or potential ALS SWM technologies, so that needs can be compared to available technologies (block 11 on Figure 1). Information from the SWM Criteria Form will also be implemented during the technology ranking step of the technology evaluation process (block 13 on Figure 1).

IDENTIFYING HIGH VALUE R\&TD - R\&TD that satisfies multiple needs can be of high value. The ALS SWM Working Group is developing requirements for a set of missions that covers a broad, yet reasonable range of locations and duration. As information on ALS R\&TD is collected and matched to Program needs, cross-cutting $R \& T D$ can be identified.

Technology development can be cross-cutting across a particular mission's requirements, meaning that the technology has the potential to satisfy more than one of the needs of that particular mission. Such a technology might also be cross-cutting across several missions, meaning that it has the potential to satisfy one or more requirements in more than one of the missions of interest. Cross-cutting technologies can be attractive to the ALS Program from the perspective of satisfying several needs with one piece of equipment. Also, research can be cross-cutting if it results in advancements in more than one technology. By funding cross-cutting R\&TD, the ALS Program could potentially get a greater benefit per dollar of investment by cutting R\&TD costs.

IMPROVED INTEGRATION - Requirements can provide a tool for improving integration between the SWM subsystem and other subsystems. For example, as missions become better defined, requirements can be written to ensure mass flow integration to and from the SWM subsystem. Thorough attention to integration assures acceptable composition of materials and rate of exchange between subsystems. For example, it could be required that materials accepted by technologies within the SWM subsystem contain certain components or are relatively free of other components. Similarly, requirements may be set so that materials passing from the SWM to other subsystems, such as the Water or Air subsystems, either contain or are free of particular components. Researchers and technology developers must be aware of these integration requirements in order to steer technology development efforts. As such, requirements definition for subsystem integration can aid in guiding R\&TD.

\section{CHARACTERISTICS OF GOOD REQUIREMENTS}

In the proceedings of the Third International Symposium of the International Council on Systems Engineering Hooks (1993) describes four qualities of a good requirement. Hooks specifies that a good requirement is clear, verifiable, attainable, and necessary. However, the development of R\&TD requirements by projecting possible mission needs is different from the development of mission requirements for existing missions. (Hooks touches on reasons for difficulty in requirements development in her 1990 AIAA paper.) Because of the unique motivations for requirements writing of the ALS SWM Working Group (as discussed in the previous section of this paper), some aspects of these four qualities may not be fulfilled in the traditional sense.

While it is possible for the ALS SWM Working Group to develop clear, verifiable requirements, the development of attainable and necessary requirements is more of a challenge and requires that assumptions be made. Such assumptions must be clearly documented in conjunction with the requirements that are produced.

Hooks states that in order for a requirement to be attainable, it must be technically feasible and fit within budget, schedule, and other constraints. However, the ALS Program performs R\&TD for future missions, thereby inherently changing technical feasibility with time. Additionally, constraints on budget, schedule and other issues (such as maximum launch mass and volume, or power and cooling availability) are not necessarily predictable. As a result, requirements that are developed for guiding R\&TD for future missions must be encompassing, so that advantageous technology options are not unintentionally excluded. In other words, requirements must be written so as to maximize (within reason) efforts for improved technological capability. This can be visualized by imagining two concentric circles, as

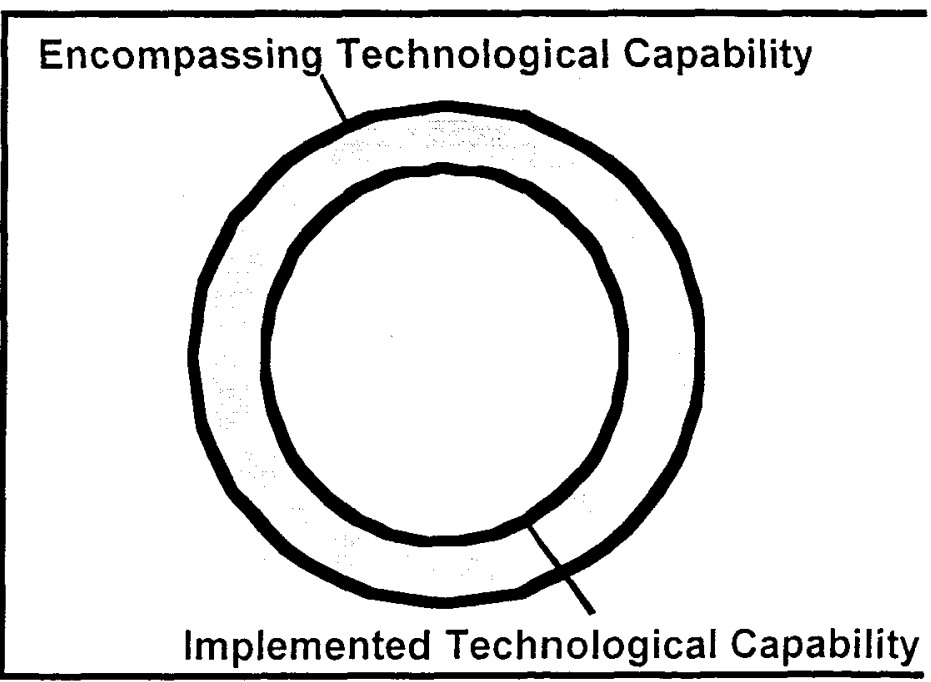

shown in Figure 2.

Figure 2. Venn Diagram of Implemented and Encompassing Technological Capability.

In Figure 2, the inner circle represents the technological capability that will be implemented in future human-rated long-duration missions. The specifics of the inner circle 
will not be defined until an actual mission is designed. In order to be prepared to meet the needs of the inner circle, a range of technological capability must be developed in advance, during the R\&TD stages of mission preparation. The outer circle represents that range of encompassing capability. Because the ALS R\&TD budget is finite, the outer circle must also be defined as a finite set of capabilities. However, the outer circle should ideally be conservative enough to encompass the inner circle. In other words, technological capability developed in the R\&TD phases of mission planning must be broad enough to enable the achievement of the actual future mission needs, yet finite enough to allow for decent progress within a finite budget. The two concentric circles in Figure 2 can also represent the mission requirements that drive the need for technological capability. This concept is discussed further in the "Requirements Forecasting" section of this paper.

In order to remain adequately encompassing in requirements writing for SWM R\&TD, development must cascade from encompassing, system level assumptions. For example, one encompassing, system-level assumption may be that the SWM subsystem will not rely upon the availability of in-situ resource utilization (ISRU) resources, such as obtaining water from regolith or $\mathrm{CO}_{2}$ for plant growth from the atmosphere during a Mars mission. The resulting requirement of such an assumption is that technologies must be developed which enable necessary resource recovery within the life support system itself. In other words, a life support system that can meet requirements without relying on ISRU is an encompassing need from the perspective of ALS R\&TD. It is critical to note that the development of life support systems that are operable without ISRU by no means suggests that ISRU technologies should not be developed. In fact, it would be ill-advised to ignore the vast, potential advantages of ISRU technologies. Systems that are able to take advantage of ISRU availability are of high priority, as are systems that can operate independently of ISRU, in case ISRU is unavailable.

It could be argued that the most encompassing approach to take in terms of promoting life support technological capability would be to assume that the life support system must be capable of complete closure, regardless of the mission location, duration or other factors. However, the system-level assumptions made by the SWM Group in developing requirements to drive R\&TD efforts must be "encompassing, yet reasonable". The Group must use sound judgement to determine what types of requirements can be reasonably expected for a range of mission options.

Because the SWM Working Group cannot address all possible system-level requirements, the Group must focus on the requirements that are suspected to have a large impact on the need for SWM R\&TD. Naturally, some requirements may be missed in the process. Also, anticipated requirements will likely change over time, as mission scenarios and technological capability change. Thus, an iterative approach to requirements development should be implemented.

\section{DRIVERS FOR LIFE SUPPORT TECHNOLOGICAL REQUIREMENTS}

In order to provide direction to SWM R\&TD by implementing the Technology Selection Process in Figure 1, attention must be paid to mission characteristics that drive the need for life support technological capability. Drivers for required technological capability can be categorized into five areas. These are illustrated in Figure 3 and listed below. These drivers must be considered in executing block 9 of Figure 1. This is just one of many acceptable ways of categorizing such drivers.

- Mission Objectives

- Mission Location, Duration and Inherent Attributes

- Crew Well-being

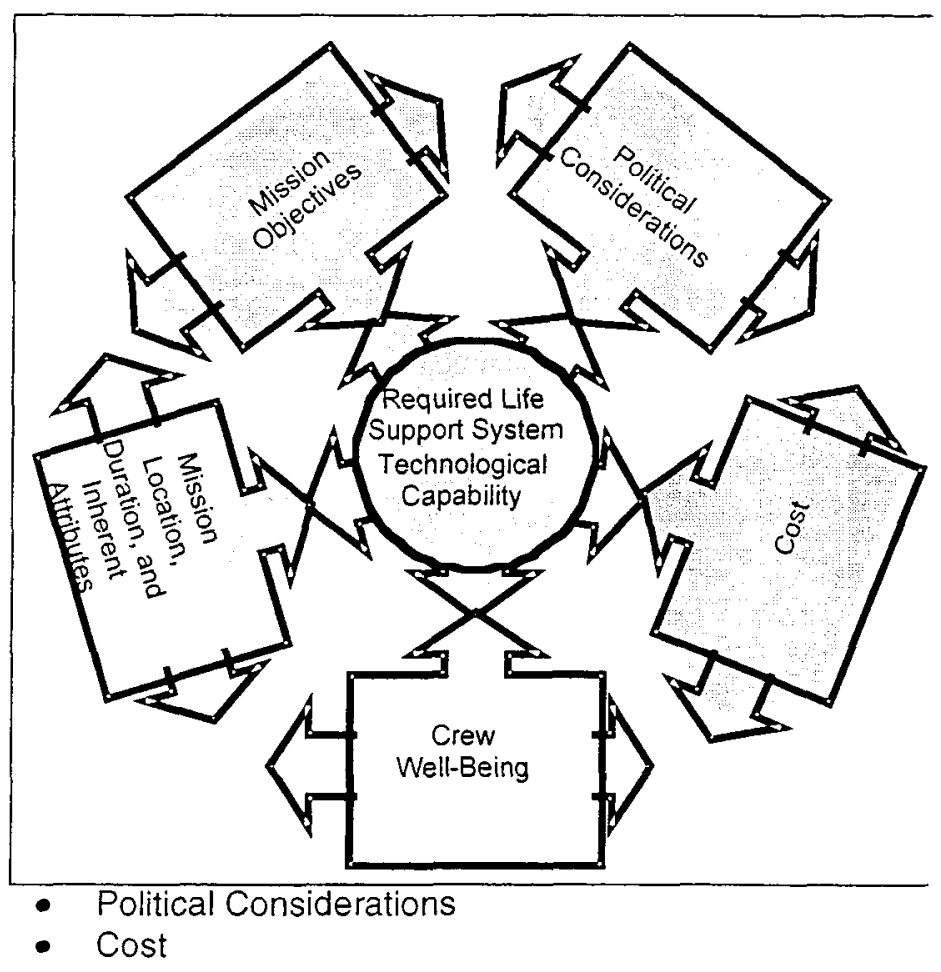

Figure 3. Requirements Drivers for Life Support System Technological Capability

It is critical to note that the five drivers of life support technological requirements are not independent of each other. In fact, it can be stated with a high degree of confidence that, for any human-rated mission, drivers of technology development will be greatly overlapping.

Obviously, the need for crew well-being drives the need for life support technological capability. The most fundamental of crew needs (acceptable atmospheric 
conditions, drinking water, food, etc.) necessitate technology for a human-rated mission. However, adjacent drivers, such as mission duration, objectives, politics and cost also come into play in defining what is an acceptable crew well-being, in terms of both physiology and psychology. For instance, as mission duration lengthens, greater demands are placed on life support technology in terms of providing the crew with a habitat that is similar in function and form to that of a dwelling on Earth.

As another example, mission location can interact with other drivers to compel needed life support capability. For instance, mission location and mission objectives are greatly interrelated and can direct the extent of extravehicular activity (EVA) during a mission. In general, the greater the EVA load, the greater technological capability that is needed to compensate for mass losses that result from EVA.

"Scientific, programmatic, or political pressures usually dictate a mission's basic requirements and constraints. Human physiology and psychology add additional requirements..." (Connolly, 1999). This quote illustrates that, although often overlooked in the very early planning stages of a mission, political considerations can have a large impact on necessary life support capability. For example, the decision to make a mission an international effort can place unexpected requirements on a life support system, thereby driving technological capability. As another example, for human-rated planetary missions, politically-driven planetary protection constraints can have a heavy impact on requirements.

Obviously, cost is an interacting factor with all drivers of technological capability. If a requirement is at all flexible, the benefit of the requirement should be weighed against the additional cost that it conveys.

\section{REQUIREMENTS FORECASTING}

The ALS SWM Working Group can forecast some requirements of future missions, based upon historical mission needs. However, the Group must make reasonable modifications to account for how future longterm missions might differ from those of the past. Because future mission requirements drivers and resultant life support $R \& T D$ needs cannot be identified with complete confidence, we must attempt to encompass those needs. Reasonably encompassing requirements can be projected, to avoid the possibility of excluding actual requirements of future missions.

The set of all possible requirements for future missions is the set of predicted requirements plus the set of unpredictable requirements. Predicted requirements are made up of inherited requirements and modified requirements. Figure 3 shows a Venn diagram of the set of possible requirements for future missions. As shown in the diagram, the predicted requirements do not perfectly overlap with the actual requirements. Actual future mission requirements will likely include some inherited requirements, some modified requirements and some unpredicted requirements. It is a goal of the SWM Working Group to try to minimize the set of unpredictable requirements, so as to maximize the preparedness of life support technology for future longterm missions.

It was previously mentioned that the two concentric circles in Figure 2 can represent the mission requirements that drive the need for technological capability. Figure 3 is therefore merely a progression of Figure 2 , as the reality of unpredictable requirements is acknowledged.

INHERITED REQUIREMENTS - Some requirements, such as those pertaining to human physiological needs, are well defined and relatively inflexible. Such requirements can be adopted fairly directly from historical requirements documents. Examples include minimum and maximum atmospheric pressures, temperature and relative humidity, as well as quantity and quality limits of food and drinking water.

MODIFIED REQUIREMENTS - The ALS SWM Group may modify requirements from previous missions, thereby carrying over known information without keeping inappropriate requirements. For missions of very long duration, even some values that are typically considered to be "fixed" (e.g. atmospheric pressure, temperature, etc.) may need to be even more rigid, thereby necessitating some modification. For example, if an equivalent probability of catastrophic failure is desired, a greater degree of system reliability would be needed for a Mars mission as compared to the ISS. (The alternative is to accept an increased level of risk.) Therefore, ISS reliability requirements might provide a guideline for the SWM Group, but more stringent modifications to those guidelines may be necessary, thereby promoting advances in life support capability.

UNPREDICTABLE REQUIREMENTS - Some requirements, such as those which may be politically driven, are not very easily predicted. Will it be required that the first human-rated mission to Mars be an international collaboration? If so, what impacts would that have on the design of the life support system? What types of social and political views will there be for planetary protection, and how will that affect the life support system design? Such non-technical issues may ultimately have real, technical influences on any aspect of the mission, including LSS design. By remaining encompassing in the requirements that are developed, the SWM Working Group hopes to minimize the impact of requirements that are unpredictable, while still maintaining a manageable, cost-effective program. 


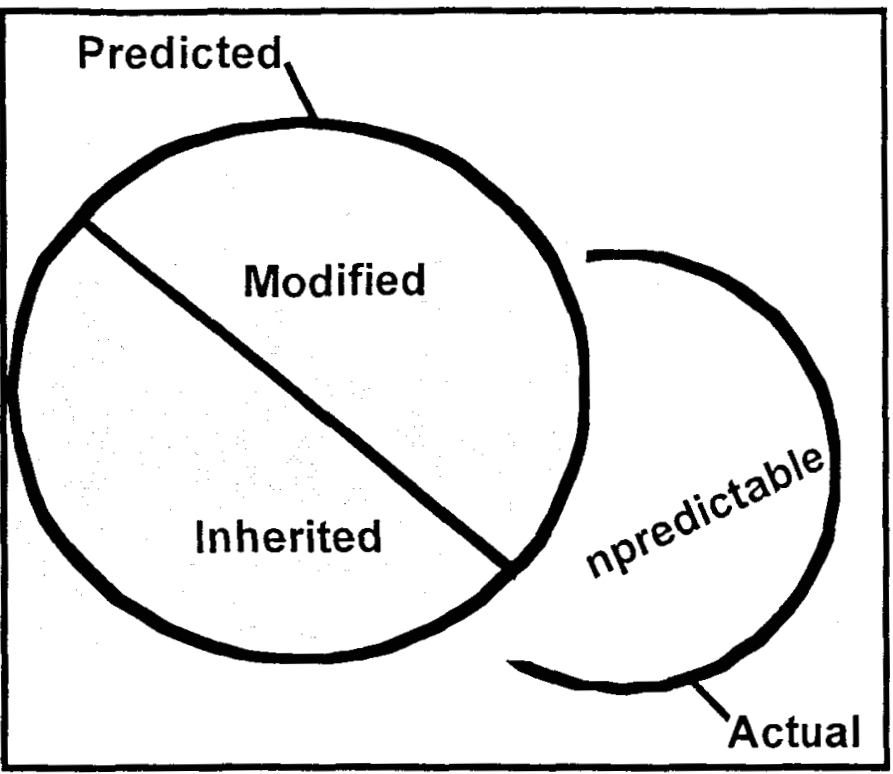

Figure 4. The set of predicted and actual future mission requirements.

\section{SUCCESSIVE MODIFICATION}

The effort of developing requirements for future missions is one of successive modification. The reason for this is three-fold:

- What is both required \& desired for missions changes with time,

- What technology is available to achieve those desires changes with time, and

- Our understanding of the optimal approaches to match the two previous, bulleted entities changes with time.

Change in what is desired can be due to the change of scientific, political or social views. Change in what is available is due to the advancement of technology as R\&TD is performed. Change in our understanding of the best way to match those two entities is due to progress in modeling and analysis. It should also be noted that there will also always be an interaction between what is desired for missions, what is available for missions and in our understanding of optimal approaches.

Accommodation for change can be viewed on both a micro-scale and a macro-scale. On a micro-scale, requirements definition for a particular mission is an iterative process until a mission is actually designed. If the cost associated with predicted, encompassing requirements is too. high, then some requirements can be modified, to a certain degree. In other words, not all requirements are absolute, as was discussed earlier in this paper. Some "requirements" are flexible and should be redefined at some points, based upon the associated cost/benefit impacts. Similarly, as non-LSS technology advances, requirements may change as well. For example, if a major advancement occurs in propulsion technology, the amount of time spent in Mars transit may change drastically. This may, in turn, greatly impact what is considered a "reasonably encompassing" need for the resultant life support technological capability.

On a macro-scale, mission priority is subject to change. For this reason, the Technology Selection Process shown in Figure 1 should be revisited, starting from the first block, as necessary. Additionally, the technology selection process itself should be modified over time, as necessary.

\section{REQUIREMENTS INTEGRATION}

It is important to consider propagation when developing requirements. In the ALS Program, the life support system is often divided into subsystems. Thus, it is natural to develop requirements on a subsystem basis. Requirements that are incompatible across subsystems will result in R\&TD that is also incompatible across subsystems. Thus, requirements must be developed using "system-level thinking", in order to assure R\&TD compatibility and to reduce risk.

In well-defined projects, requirements should be developed using a system-level methodology (top-down approach). In such projects, some requirements lie at the system-level, meaning that those requirements are achieved by collaborating requirements among several subsystems. Therefore, for well-defined projects, it is possible to determine the cost-effectiveness of achieving requirements through integration between several subsystems, versus the cost-effectiveness of achieving the requirements within one subsystem.

Because the ALS Program performs R\&TD for many unscheduled missions that are inherently ill-defined, it is difficult to develop compatible, cross-subsystem requirements. Many ALS technologies are at a low level of technology readiness level (TRL) for spaceflight. As a result, further research may be needed to understand the extent of the flexibility of low TRL technologies. Consequently, the most cost-effective method of satisfying requirements may need to be determined by studying several different approaches simultaneously.

For example, a mission need may be that human pathogens in solid waste materials be destroyed, to a certain degree of confidence. This may mean that all materials that have a high potential to contain human pathogens (such as toilet wastes, hygiene wastes, and food wastes) be treated for pathogen destruction. Some SWM technologies for destroying human pathogens (such as composting) are incompatible with some of the metallic materials used in food packaging. Therefore, R\&TD would be required to develop or modify technologies to be able to treat such food packaging materials (such as physicochemical technologies). Similarly, requirements that are developed within the food subsystem should be encompassing and account for integration with other subsystems. If encompassing 
requirements were developed by both subsystems of a particular interface, then such dilemmas would be researched simultaneously on both ends. As a result, as time progresses, it may be determined that it is more cost-effective for the food subsystem to develop nonmetallic packaging materials than it is for the SWM subsystem to develop technologies to treat such materials, or vice versa.

As another interface example, incinerator off-gases may require treatment for contaminant removal before being vented to the crew cabin. This challenge can be solved either through R\&TD of alternative incinerator design or through R\&TD within the Air subsystem for removal of incinerator-specific gas contaminants. The most encompassing approach, for increased assurance of satisfying actual mission requirements, is to work the problem from both ends. Thus, at this early stage of mission definition, it is perfectly acceptable to have redundant requirements between subsystems.

In order to ensure that needed capability is satisfied in a cost-effective manner, requirements satisfaction should be investigated from several angles. As time passes and the certainty in mission definition increases, requirements that are very difficult to meet or are expensive to satisfy within the SWM subsystem can be modified by imparting constraints that propagate to other subsystems.

\section{CONCLUSION}

This paper discussed the following points:

1. Motivations for developing ALS SWM requirements include providing ALS SWM researchers and technology developers with goals and guidelines, matching Program needs to available technologies, identifying high-value $R \& T D$, and improving integration between and within subsystems.

2. Requirements for ill-defined missions are inherently tied to assumptions. Such requirements should also be encompassing, iterative, and developed with a system-level perspective.

3. Drivers for life support technological requirements include mission objectives, mission location, duration and inherent attributes, crew well-being, political considerations, and cost.

4. Some requirements of future missions are unpredictable.

5. The set of encompassing requirements that is developed in the very early stages of mission concept development can be inherited from historical requirements, or modified (derived) from historical requirements.

6. The effort of developing requirements for future missions is one of successive modification. The reason for this is three-fold: change in what is required/desired for missions, change in what technology is available to achieve those desires, and change in our understanding of the optimal approaches to match those two entities.

7. At the early stages of mission development, it is perfectly acceptable to have redundant requirements among subsystems. This encourages R\&TD from all perspectives of a requirement.

\section{ACKNOWLEDGMENTS}

The work presented in this report is sponsored by NASA partially through Contract number NAS9-19100 and partially through the New Jersey NASA Specialized Center of Research and Training (NJ-NSCORT). The authors would also like to acknowledge the researchers in the ALS SWM Working Group, who have contributed to the SWM R\&TD Plan and requirements development process.

\section{REFERENCES}

1. Alazraki, M.P. (2001) Advanced Life Support Solid Waste Management Research and Technology Development Plan, NASA Johnson Space Center, Houston, TX.

2. Alazraki, M.P.; Drysdale, A.E.; Fisher, J.W.; Hogan, J.A.; Levri, J.A. (2002) Solid Waste Management Requirements Definition for Advanced Life Support Missions - Results. $32^{\text {nd }}$ International Conference on Environmental Systems, SAE Technical Paper \#2002-01-????.

3. Connolly, John F. (1999) Mars Design Example, in Human Spaceflight, edited by Larson, W.J. and Pranke, L.K., The McGraw-Hill Companies, Inc., New York.

4. Hogan, J.A.; Fisher, J.W.; Alazraki, M.P.; Levri, J.A. (2002) Considerations in Selection of Solid Waste Management Approaches in Long-Duration Space Missions. $32^{\text {nd }}$ International Conference on Environmental Systems, SAE Technical Paper \#2002-01-????.

5. Hooks, IVy (1990) Why Johnny Can't Write Requirements. AIAA Conference.

6. Hooks, Ivy (1993) Writing Good Requirements. Proceedings of the Third International Symposium of the International Council On Systems Engineering (INCOSE), Volume 2.

7. Levri, J.A.; Hogan, J.A.; Alazraki, M.P. (2001) Evaluation Criteria for Solid Waste Management Research and Technology Development, American Society for Gravitational and Space Biology (ASGSB) $17^{\text {th }}$ Annual Meeting, Alexandria, Virginia, November 7-11.

8. Stafford, K.W.; Jerng, L.T.; Drysdale, A.E.; Maxwell, S.; Levri, J.A. (2001) "Advanced Life Support Systems Integration, Modeling, and Analysis Reference Missions Document," edited by Ewert, M.K. and Hanford. A.J., JSC-39502, Revision A, NASA Johnson Space Center, Houston, Texas. 


\section{CONTACT}

Julie A. Levri

Mail Stop 239-8

NASA Ames Research Center

Moffett Field, CA 94035

(650) 604-6917 (telephone)

(650) 604-1092 (fax)

ilevri@mail.arc.nasa.gov

\section{DEFINITIONS, ACRONYMS,} ABBREVIATIONS

ALS: Advanced Life Support
EVA: Extra-Vehicular Activity

INCOSE: International Council on Systems Engineering

ISS: International Space Station

LSS: Life Support System

RMD: Reference Missions Document

R\&TD: Research and Technology Development

SWM: Solid Waste Management

TRL: Technology Readiness Level 